\title{
The effect of sulforaphane on oxidative stress and inflammation in rats with toxic hepatitis induced by acetaminophene
}

\author{
Dokumacioglu $\mathrm{E}^{1}$, Iskender $\mathrm{H}^{1}$, Aktas $\mathrm{MS}^{2}$, Hanedan $\mathrm{B}^{2}$, Dokumacioglu $\mathrm{A}^{3}$, Sen $\mathrm{TM}^{4}$, Musmul $\mathrm{A}^{5}$ \\ Department of Nutritition and Dietetics, Faculty of Healthy Sciences, Artvin Coruh University, Artvin, Turkey. \\ edadokumacioglu@yahoo.com
}

\section{ABSTRACT}

OBJECTIVE: The aim of the present study was to reveal the possible effect of sulforaphane on oxidative stress and inflammation in rats liver with toxic hepatitis induced by acetaminophene.

BACKGROUND: Sulforaphane is a compound with high antioxidant properties. Acetaminophen, which is a para-aminophenol derivative, can lead to fatal hepatic necrosis with direct hepatotoxic effects at high doses. METHODS: Thirty six male Sprague-Dawley rats were randomly divided into four groups. Control group $(n=9)$ was fed with standard rat chow and water for 3 days. Group APAP $(n=9)$ received a single dose acetaminophen $1 \mathrm{~g} / \mathrm{kg}$ by oral gavage in addition to standard chow and water. Group SFN $(n=9)$ received sulforaphane $500 \mu \mathrm{g} / \mathrm{kg}$ by oral gavage in addition to standard chow and water for 3 days. Group APAP+SFN $(n=9)$ received sulforaphane $500 \mu \mathrm{g} / \mathrm{kg}$ and a single dose acetaminophen $1 \mathrm{~g} / \mathrm{kg}$ by oral gavage in addition to standard chow and water. Acetaminophen was administered three hours after SFN administration.

RESULTS: Neopterin, MDA, AST, ALT and CRP levels of group APAP were significantly increased compared to control group. GSH level of group APAP was significantly lower than in the control group.

CONCLUSION: Sulforaphane is a protective agent against acetaminophen-induced liver damage and it can be added in the treatment protocol (Tab. 1, Fig. 5, Ref. 51). Text in PDF www.elis.sk.

KEY WORDS: acetaminophen, lipid peroxidation, neopterin, oxidative stress, sulforaphane.

\section{Highlights}

- The analgesic acetaminophen causes a potentially fatal hepatotoxicity when taken in overdose.

- Sulforaphane has a protective effect on the liver.

- SFN has preventive action in lipid peroxidation on liver injury.

- Neopterin is a biomarker for the animal model of APAP-induced liver injury.

\section{Introduction}

Due to its strategic role in the body, the liver is a key organ for metabolism, secretion and excretion of various xenobiotics, environmental pollutants and chemotherapeutic agents that the body is exposed to. Liver damage and subsequent deaths remain to be

${ }^{1}$ Department of Nutritition and Dietetics, Faculty of Healthy Sciences, Artvin Coruh University, Artvin, Turkey, ${ }^{2}$ Department of Internal Medicine, Faculty of Veterinary Medicine, Ataturk University, Erzurum, Turkey, ${ }^{3}$ Department of Medical Biochemistry, Hopa Government Hospital, Artvin, Turkey, ${ }^{4}$ Department of Medical Biochemistry, Faculty of Medicine, Karadeniz Teknik University, Trabzon, Turkey, and ${ }^{5}$ Department of Biostatistics, Faculty of Medicine, Eskisehir Osmangazi University, Eskisehir, Turkey

Address for correspondence: E. Dokumacioglu, Dr, Artvin Coruh University, Faculty of Healthy Sciences, Department of Nutrition and Dietetics, Artvin, Turkey.

Phone: +466.2121301 .2144$

Acknowledgments: This work was supported by Coordinator of Scientific Research Projects (2014.M80.02.04) at Artvin Coruh University. a common concern worldwide $(1,2)$. Hepatotoxicity as a result of unconsciously used drugs is one of the common causes of acute liver damage. Studies have shown that $58 \%$ of acute liver failure is caused by drugs, and acetaminophen (APAP) takes a significant place among these drugs (3).

Acetaminophen, which is a para-aminophenol derivative, can lead to fatal hepatic necrosis with direct hepatotoxic effects at high doses. For individuals with alcohol abuse and malnutrition, the use of acetaminophen even at treatment doses may cause asymptomatic transferase elevation or hepatotoxicity. Acetaminophen toxicity impairs the mechanism of liver detoxification. Glutathione (GSH) stores become inadequate when taken in overdose and $\mathrm{N}$-acetyl-p-benzoquinone (NAPQI) which is a toxic metabolite of acetaminophene causes hepatocellular necrosis by binding to the cytoplasmic proteins $(3,4,5)$. Although the exact mechanism of the hepatic damage is not fully clarified, oxidative damage is the most underlined factor. Increased oxidative stress causes impairment of intracellular calcium homeostasis, signal transduction and mitochondrial permeability, and eventually ceases production of adenosine triphosphate (ATP). As a result of all these factors, cell necrosis occurs $(4,6)$. Malondialdehyde (MDA) is the most commonly used marker for oxidative damage. MDA, the end product of lipid peroxidation, affects ion exchange in the membrane by increasing cell membrane permeability. As a result, intracellular ion balance is disturbed, causing impairment of enzyme activities. It leads to tissue damage both in its own region of formation and in further remote regions that it diffused to (7). 
It is not always easy to predict hepatotoxicity because it exhibits variations depending on the sensitivity of the person as well as the drug used. Therefore, early diagnosis is very important in hepatotoxicity to achieve a more effective treatment. C-reactive protein (CRP) is an acute-phase reactant that is mainly synthesized by hepatocytes in the liver as part of an inflammatory response. CRP plays an important role in the inflammatory process, and as such could potentially be affected by liver disease (8). Neopterin, a pyrazinopyrimidine compound, is used as a very popular biomarker especially in important pathologies where cellular immunity mechanisms are actived. It is remarkable because it is easily measurable and it serves as a guide in the evaluation of diagnosis, prognosis and treatment efficacy $(9,10)$. Various studies reported that neopterin can be used as an important biomarker in many systemic toxicities and pathologies and as a new biomarker in liver diseases $(11,12)$. Many studies indicated that neopterin was associated with interactions between both reactive oxygen metabolites and oxidative stress. Neopterin plays a role in the defense system by increasing the cytotoxic effects of reactive oxygen metabolites that are formed in the body against invasive pathogens. The increase in serum levels of neopterin along with the increase in severity of inflammation, infection, and malignancy indicate that neopterin is part of the defense system $(6,13)$.

Antioxidant defense mechanisms are developed in the body to prevent the formation and damage of free radicals. In the case of hepatotoxicity, free radical production increases and antioxidant defense mechanisms become insufficient (6). Sulforaphane (SFN) is a compound with high antioxidant properties present in broccoli. Recent studies report that sulforaphane is safe and tolerable and exhibits its beneficial effects by activating liver phase II detoxification enzymes. Sulforaphane is considered as a potent fighter against oxidative stress with its antioxidant, anti-inflammatory and anti-tumor effects $(13,14)$. In this study, we intended to investigate whether sulforaphane is protective against acetaminopheninduced liver damage or not, by evaluating the levels of neopterin, a cellular immunosystem activation marker, as well as CRP, lipid peroxidation and glutathione levels.

\section{Materials and methods}

\section{Chemicals}

APAP and SFN were purchased from Sigma Chemical Co (St. Louis, MO, Germany).

\section{Experimental animals}

Randomly selected 36 male Sprague-Dawley rats were used in the study, each about $200-250 \mathrm{~g}$ and 6-8 weeks of age. The animals were housed in special rooms with ambient temperature of $22 \pm 2{ }^{\circ} \mathrm{C}$ and humidity of 50-60\%, under photoperiod of 12 : $12 \mathrm{~h}$ light: dark and were given tap water and standard diet. This study was carried out in the Atatürk University's Experimental Animal Laboratory of the Medical and Experimental Application and Research (ATADEM) in accordance of the Atatürk University's Local Ethical committee decision of date 30.04.2014 and the project number of 2014/63.

\section{Experimental groups}

The study consisted of four groups, each including nine rats, namely control group, acetaminophen (APAP) group, sulforaphane (SFN) group and APAP+SFN group. Experimental studies continued for three days. Nothing was given to the control group throughout the experiment. SFN was administered for treatment via oral gavage at a dose of $500 \mu \mathrm{g} / \mathrm{kg}$ for three days to the rats of $\mathrm{SFN}$ and APAP $+\mathrm{SFN}$ groups (15). Acetaminophen was dissolved in hot saline and administered on the third day to produce toxic hepatitis via a single oral gavage at a dose of $1 \mathrm{~g} / \mathrm{kg}$ to the rats of the APAP and APAP+SFN groups (16). APAP was administered three hours after SFN administration in the APAP+SFN group.

\section{Preparation of serum and tissue samples}

At the end of the experiment, the rats were anesthetized by intraperitoneal injection of a mixture of ketamine hydrochloride (60 $\mathrm{mg} / \mathrm{kg})$ and xylazine $(12 \mathrm{mg} / \mathrm{kg})$ and sacrificed 24 hours after the last administration. Animals were anesthetized by intraperitoneal injection of a mixture of ketamine hydrochloride $(60 \mathrm{mg} / \mathrm{kg})$ and xylazine $(12 \mathrm{mg} / \mathrm{kg})$. Their blood was collected into biochemical tubes by heart puncture. Blood samples were centrifuged for 10 minutes at $3500 \mathrm{rpm}$ to obtain a clear serum, which was stored at $-80{ }^{\circ} \mathrm{C}$. Serum samples were used to determine the activities of aspartate aminotransferase (AST), alanine aminotransferase (ALT) and CRP. The liver tissue was excised immediately and washed with icecold saline. Liver tissues were homogenized with $0.1 \mathrm{M}$ phosphate buffer at $\mathrm{pH}$ 7.4. (17). The homogenates were used to determine the total protein, MDA, GSH and neopterin levels.

\section{Biochemical analyses}

Hepatotoxicity was assessed by quantifying the serum activities of AST and ALT. AST, ALT and CRP levels were measured using a modular autoanalyzer (Roche, Mannheim, Germany). The results of AST and ALT were expressed as U/L. The results of CRP was expressed as mg/dl. Hepatic MDA levels were measured spectrophotometrically based on the method described by Ohkawa et al (18). Then, $0.5 \mathrm{~mL}$ of $8.1 \%$ sodium dodecyl sulfate (SDS), $0.5 \mathrm{~mL}$ of $0.8 \%$ thiobarbituric acid (TBA), $1.0 \mathrm{~mL}$ of 10 $\%$ of trichloracetic acid (TCA), $1.0 \mathrm{~mL}$ of $2 \%$ glacial acetic acid/ sodium hydroxide $(\mathrm{NaOH})(\mathrm{pH}=3.5)$ and $50 \mu \mathrm{L}$ of $2 \%$ butylhydroxytoluene (BHT) were added to the samples $(1.0 \mathrm{~mL})$ and this mixture was thoroughly mixed and kept in a water-bath at $95{ }^{\circ} \mathrm{C}$ for 60 mins. After the tubes had been chilled, a mixture of $4.0 \mathrm{~mL}$ of butanol/pyridine $(1: 15)$ was added and the tubes were centrifuged at $4000 \times \mathrm{g}$ at $+4{ }^{\circ} \mathrm{C}$ for 10 mins. After centrifugation, the upper organic phase was removed and the absorbance was read at $532 \mathrm{~nm}$ for all samples. MDA results were expressed as nmol/ mg protein. Hepatic GSH levels were also measured spectrophotometrically based on the method described by Beutler et al (19). The spectrophotometric assay method for glutathione (GSH) involved oxidation of GSH by the sulfhydryl reagent 5,5'-dithiobis (2-nitrobenzoic acid) (DTNB) to form the yellow derivative 5'thio-2-nitrobenzoic acid (TNB), measurable at $412 \mathrm{~nm}$. GSH results were expressed as nmol/mg protein. Tissue protein analysis was done by the method described by Lowry et al (20). Hepatic 
Tab. 1. Biochemical parameters of the groups.

\begin{tabular}{|c|c|c|c|c|}
\hline & Control & APAP & SFN & APAP+SFN \\
\hline $\operatorname{AST}(\mathrm{U} / \mathrm{L})$ & $69.33 \pm 7.80$ & $142.33 \pm 6.94^{\mathrm{a}}$ & $75.18 \pm 8.64^{d}$ & $82.25 \pm 9.18^{b, c}$ \\
\hline ALT (U/L) & $34.00 \pm 2.87$ & $95.00 \pm 6.25^{\mathrm{a}}$ & $43.67 \pm 4.76^{\mathrm{d}}$ & $53.61 \pm 5.21^{\mathrm{b}, \mathrm{c}}$ \\
\hline $\mathrm{CRP}(\mathrm{mg} / \mathrm{dl})$ & $3.42 \pm 0.10$ & $15.61 \pm 2.14^{\mathrm{a}}$ & $3.88 \pm 1.13^{\mathrm{d}}$ & $6.52 \pm 0.98^{\mathrm{b}, \mathrm{c}}$ \\
\hline GSH(nmol/mg protein) & $4.30 \pm 0.18$ & $2.45 \pm 0.54^{\mathrm{a}}$ & $4.22 \pm 0.10^{\mathrm{d}}$ & $4.19 \pm 0.20^{\mathrm{b}}$ \\
\hline MDA (nmol/mg protein) & $9.90 \pm 2.27$ & $17.92 \pm 4.24^{\mathrm{a}}$ & $10.83 \pm 2.11^{\mathrm{d}}$ & $9.79 \pm 3.10^{\mathrm{b}}$ \\
\hline
\end{tabular}

Data were given as mean $\pm \mathrm{SD}$, AST - Aspartate aminotransferase, ALT - Alanine aminotransferase, APAP - Acetaminophene, CRP - C-reactive protein; GSH - Glutathione,

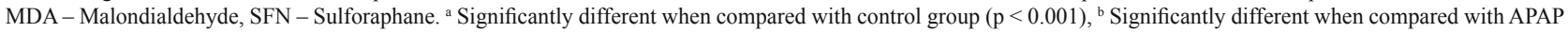
group $(\mathrm{p}<0.001),{ }^{\mathrm{c}}$ Significantly different when compared with control group $(\mathrm{p}<0.05),{ }^{\mathrm{d}}$ Significantly different when compared with control group $(\mathrm{p}>0.05)$

neopterin levels were measured by using the enzyme-linked immunoassay (ELISA) method using a rat ELISA kit (Shangai Yehua Biological Technology Co., Ltd., Shanghai, China) according to the manufacturer's recommended test procedure. Neopterin results were expressed as $\mathrm{nmol} / \mathrm{mg}$ protein.

\section{Tissue histology}

Samples of liver tissue from each animal were fixed in $10 \%$ formalin, dehydrated in ascending grades of alcohol and embedded in paraffin. Slides were examined under the microscope to detect histologic changes subsequent to staining with haematoxylin and $\operatorname{eosin}(\mathrm{H} \& \mathrm{E})$.

\section{Statistical analysis}

All data analyses were performed by using Statistical Package for the Social Sciences (SPSS) for Windows 19.0 package program (SPSS Inc., Chicago, IL, USA). Kolmogorov-Smirnov test was done to evaluate the distribution of variables. Continuous normally distributed measurements were compared across the groups by using one-way analysis of variance (ANOVA) with the Tukey method and the Student-Newman-Keuls method multiple comparisons. The descriptive statistics were demonstrated by $n$ (i.e., the sample size) and by the mean and standard deviation for continuous variables. A $\mathrm{p}<0.05$ was accepted as significant.

\section{Results}

\section{Biochemical results}

Serum AST, ALT and CRP levels were determined to increase significantly in the group of acetaminophen in comparison to the control group (respectively, $\mathrm{p}<0.001$ ) (Tab. 1). In the APAP+SFN group however, AST, ALT and CRP levels were observed to decrease statistically more significantly than in the APAP group ( $p$ $<0.001$ ), it was observed that they are significantly higher than those in the control group $(\mathrm{p}<0.05)$. No statistically significant difference was determined in terms of AST, ALT and CRP levels in SFN group when compared to the controls $(\mathrm{p}>0.05)$.

\section{Glutathione levels}

GSH levels showed a statistically significant decrease in the APAP group compared to the control group $(\mathrm{p}<0.001)$. Hepatic GSH levels in the APAP+SFN group were found to increase with respect to the APAP group $(\mathrm{p}<0.001)$ while there was no significant difference with respect to the control group ( $p>0.05)$. However, there was no statistically significant difference in the

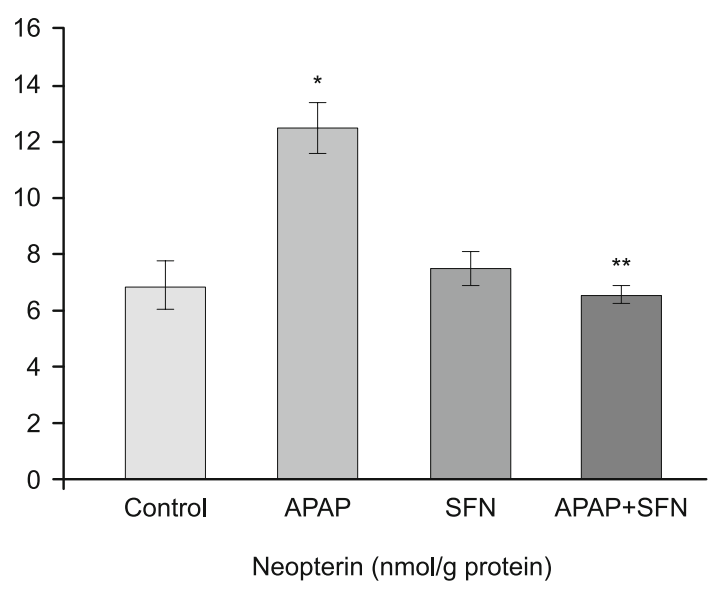

Fig. 1. Liver neopterin levels in experimental groups. * - significantly different when compared with control group $(p<0.001), * *-$ Significantly different when compared with APAP group, $(p<0.001)$.

hepatic GSH levels of SFN group in comparison to the control group $(\mathrm{p}>0.05)$.

\section{Lipid peroxidation}

Hepatic MDA levels in the APAP group increased significantly when compared to the controls $(\mathrm{p}<0.001)$. In comparison to the APAP group, MDA levels showed a decrease in the APAP+SFN group $(\mathrm{p}<0.001)$. There was no statistically significant difference in the hepatic MDA levels of the APAP+SFN group with respect to the control group $(p>0.05)$. Hepatic MDA levels of the SFN group showed no statistically significant difference when compared to the control group $(\mathrm{p}>0.05)$.

\section{Neopterin levels}

In our study, neopterin levels showed a significant increase in the APAP group with respect to the control group $(p<0.001)$ whereas neopterin levels in APAP+SFN group were observed to decrease significantly with respect to APAP group $(\mathrm{p}<0.001)$ (Fig. 1). Neopterin levels of the APAP + SFN group showed no statistically significant difference with respect to the control group $(p>0.05)$. There was no statistically significant difference in neopterin levels of the SFN group when compared to the control group $(p>0.05)$.

\section{Histology analysis}

The livers of the control and SFN groups showed normal histological structure. The liver tissues showed portal triad, and normal 
arrangement of hepatocytes with nuclei (Figs 2 and 3). APAP overdose caused marked liver damage in the form of intense cellular degeneration, sinusoidal dilatation and cellular inflammation of hepatocytes with vascular congestion (Fig. 4). APAP + SFN group

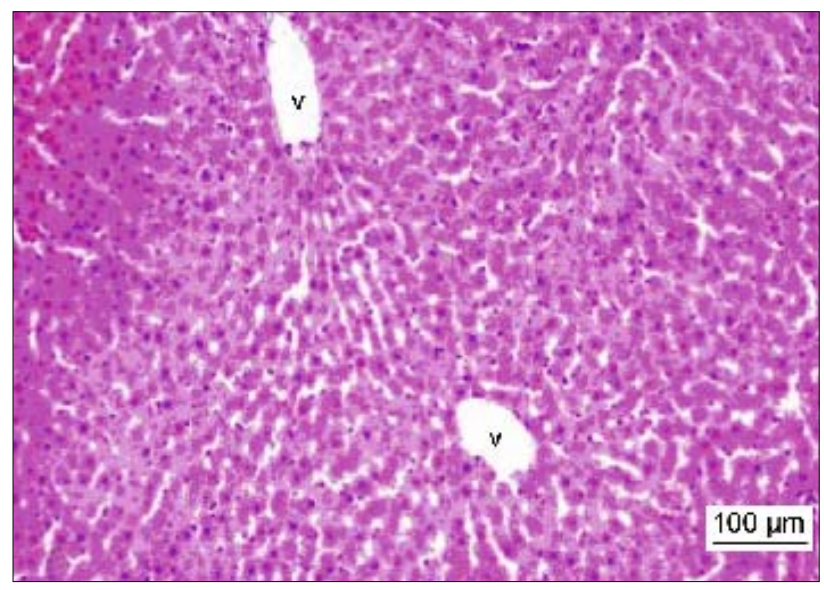

Fig. 2. Photomicrographs of rat liver (H and E, x100) from: control group showing normal hepatic architecture.

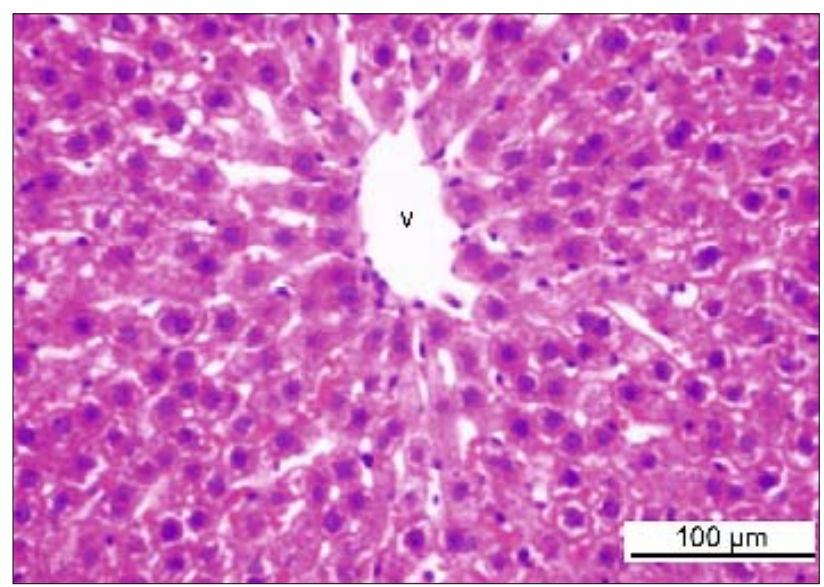

Fig. 3. Photomicrographs of rat liver (H and E, x100) from: sulforaphane group showing normal hepatic architecture.

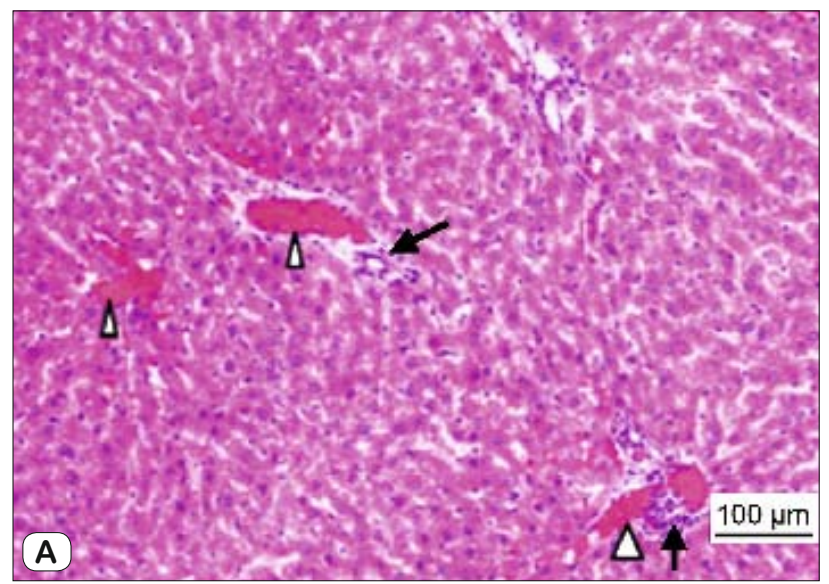

showed mild cellular inflammation and the area of liver damage was reduced compared with APAP group. SFN treatment caused significant changes in the liver histology and showed portal triad, normal arrangement of hepatocytes with nuclei and normal hepatic architecture (Fig. 5).

\section{Discussion}

One of the most important health problems on which the researchers and pharmaceutical industry collaborate globally is the drug-induced liver diseases, and they are thought to be the result of direct hepatotoxicity and adverse immunological reactions. Understanding the mechanisms of action of hepatotoxicity in relation with mortality is important for biological tracing of toxic compounds and developing treatment modalities. Drug-induced hepatotoxicity presents with a broad clinical spectrum, from mild biochemical abnormalites to acute hepatic failure $(21,22)$.

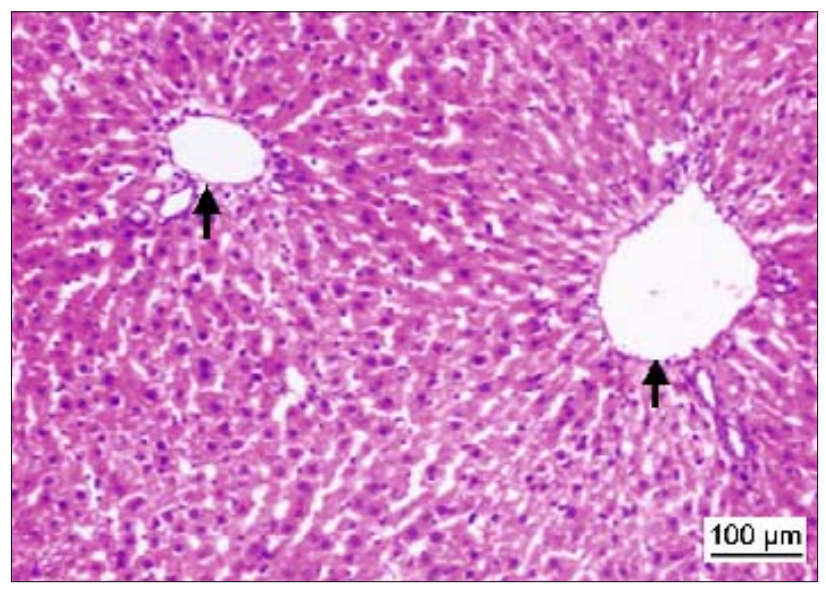

Fig. 5. Photomicrographs of rat liver (H and E, x100) from: acetamiophen plus sulforaphne group showing mild cellular inflammation and normal arrangement of hepatocytes with nuclei, and showing normal hepatic architecture. The area of liver damage was reduced compared with acetaminophen group.

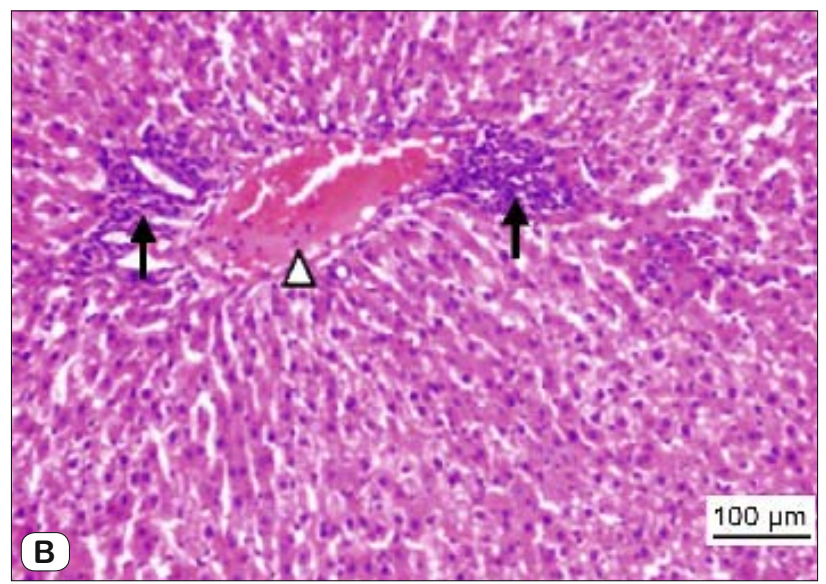

Fig. 4. Photomicrographs of rat liver (H and E, x100) from: (a) acetaminophen group showing intense cellular degeneration, sinusoidal dilatation and (b) cellular inflammation of hepatocytes with vascular congestion. 
APAP-induced hepatotoxicity is one of the leading causes of acute liver failure today. As the frequency of APAP use increases, the incidence of acute hepatic failure and mortality in overdosage is observed to increase as well (22). AST and ALT, which are key parameters of hepatocellular damage, are important routine hepatic tests assessing the presence and characteristics of the liver disease and the response to treatment (23). In our study, serum AST and ALT levels, which are indicative of hepatocellular damage, increased significantly in the toxic hepatitis group induced by APAP when compared to the control group. Sulforaphane however, was observed to reduce serum AST and ALT levels significantly compared to the toxic hepatitis group. Many different medications can cause drug-induced liver diseases. Painkillers that contain acetaminophen are a common cause of liver inflammation. These medications can damage the liver when taken in doses that are not much greater than the recommended dose (24). Inflammation plays a critical role in liver disease via induction of cellular injury and CRP plays an important role in the inflammatory process (8). In our study, serum CRP levels, which are indicative of inflammatory response, increased significantly in the hepatotoxicity group induced by APAP when compared to the control group. Sulforaphane however, was observed to reduce serum CRP levels significantly compared to the APAP-induced toxic hepatitis group. In this study, APAP-induced hepatotoxicity was evidenced by biochemical measurements and histopathological changes that coincided with the observations of other investigators.

Glutathione is a potent antioxidant highly found in hepatocytes, with a remarkable ability for free-radical scavenging in order to maintain cellular integrity and contribute to intracellular defense (25). Studies have reported that acetaminophen toxicity significantly reduced hepatic GSH levels $(26,27)$. It was shown that reduction in intracellular GSH levels alone did not cause cell death by itself, but the depletion of GSH levels was determined to trigger the cytotoxicity (28). It was reported that reduction in GSH levels can cause formation of reactive oxygen species and mitochondrial dysfunction. In acetaminophen-induced hepatotoxicity, the depletion of GSH that can detoxify NAPQI reactive metabolites is thought to play a role in the onset of liver damage $(6,29)$.

Antioxidants have a significant protective role in the acetaminophen-induced liver damage (25). Sulforaphane is one of the natural antioxidants that has an effect on important cellular defense systems (30). Santana-Martínez et al (31). It is reported that sulforaphane significantly increased the GSH levels and plays a protective role against the oxidative stres. Also in our study, in line with the results in literature, we determined that sulforaphane had a protective role against acetaminophen-induced hepatotoxicity and GSH levels significantly increased in the treatment group compared to the group with APAP-induced toxicity. GSH plays a key role in cellular defense systems. Dysregulation of GSH homeostasis plays a role in the etiology and progression of many diseases including cancer, aging, inflammatory and neurodegenerative diseases (32, 33). In their study, Baek et al (33) reported the protective effects of sulforaphane on $\mathrm{CCl}_{4}$-induced liver damage, and indicated that such effects were mediated by the induction of liver phase II enzymes. Likewise, in another study, increasing hepatic GSH levels were shown to be protective against hepatic damage in the group treated with sulforaphane before liver ischemia reperfusion (Zhao et al, 2010). Considering our results as well as those found in literature, we can say that sulforaphane protects the liver excellently by inducing GSH, which is a powerful cellular defense system against possible damages from various substances.

Oxygen free radicals and lipid peroxidation, which play a role in the pathogenesis of various hepatic diseases, exhibit their direct damage by causing changes in membrane structure and indirect damage by leading to the formation of reactive aldehydes (34, 35 ). As a result of such deterioration in the membrane structure, some changes occur in membrane functions such as ion transport and enzyme activity. Free radicals and lipid peroxides are admitted to play a role in the development of various diseases such as diabetes, atherosclerosis, cancer and liver cirrhosis, while lipid peroxidation is suggested to be closely related to the tissue damage from acetaminophen-induced hepatotoxicity $(36,37)$. In addition, the superoxide radical and singlet oxygen are reported to induce liver damage through lipid peroxidation (38). Recent experimental hepatotoxicity studies demonstrated increased MDA levels in plasma and liver tissue $(39,40)$. The 4-hydroxynonenal molecule, which is a lipid peroxidation product and also an aldehyde, is reported by Terneus et al (41) to be linked to liver tissue proteins in acetaminophen-induced groups. Based on our findings and those found in literature; we can claim that increased MDA levels in acetaminophen-induced toxic hepatitis is one of the consequences of lipid peroxidation caused by free radicals. This result suggests that free radicals and oxidative stress play a role in tissue damage in toxic hepatitis. Increased lipid peroxidation products, especially aldehydes, can interact with various biomolecules, affecting their functions. In the light of the literature, increased MDA levels in the liver tissue in our study suggest that MDA will continue to have adverse effects both in the region it is formed and in remote tissues.

All living organisms are constantly exposed to free radicals, both physiologically and through external factors. Free radical formation and lipid peroxidation is either a normal physiological state or a signal of disease development. The human body neutralizes the oxidants by innate antioxidant defense mechanisms. However, in some cases innate antioxidant defense is not adequate. Exogenous antioxidants must be additionally taken $(42,43)$. To the best of our knowledge, there are very few studies investigating the effects of sulforaphan on acetaminophen-induced hepatotoxicity (26). Gaona-Gaona et al (15) experimentally induced liver damage and reported that sulforaphane reduced the MDA levels in the liver by decreasing lipid peroxidation. In our study, we determined that sulforaphane significantly decreased liver MDA levels in the treatment group compared to the hepatotoxicity group. The decreased MDA levels in the treatment group may suggest that sulforaphane can reduce oxidative stress-mediated liver damage and distal tissue damage. As a result, many diseases can be prevented and the duration and quality of life can be improved by avoiding free radicals and taking antioxidant-rich nutrients.

Recent studies indicated that serum or urine neopterin levels can be used as diagnostic and prognostic markers in various pathologic conditions $(44,45)$. Abnormal neopterin concentrations are shown 
453-459

to be detected in body fluids in various clinical situations, they are also reported to provide information about the current state of the cellular immune response and usually helpful in predicting disease progression (46). Increased neopterin levels indicate the endogenous formation of gamma interferon and thus, monitoring neopterin levels allows the activation status of the cell-mediated immune system to be determined. Some xenobiotics, various viral, bacterial, inflammatory and malign diseases, stressful factors such as physical changes and various infections can result in increased neopterin concentrations of biological fluids such as urine and serum (47).

Macrophages as well as inflammatory cytokines were reported in various studies to increase in the liver in acetaminophen-induced hepatic damage $(48,49)$. There is an inceasing interest towards neopterin in recent years, which is regarded as a biochemical marker of cell-mediated immune response. So, in this study, based on the fact that neopterin is a marker of cellular immune response, we investigated the value of neopterin in early diagnosis of drug-related toxic hepatitis and whether it might be a criterion for initiating the treatment by determining neopterin levels, or not. Demirbas et al (11) reported increased neopterin levels in acetaminopheneinduced liver damage and argued that neopterin levels can be used as a new marker due to its preclinical features in these cases. In another study conducted by Cakir et al (48), nephrotoxicity was established by acetaminophen and neopterin levels were reported to be high. In adult patients with liver cirrhosis, Fernandez et al (50) found higher serum neopterin levels compared to the healthy control group. In parallel with the literature, we also found that neopterin levels were higher in the APAP-induced hepatotoxicity group with respect to the control group. Based on the literature and our data, as a sensitive indicator of cellular immunological activation of various diseases, increased levels of neopterin may be one of the factors responsible for the progression of APAP-induced hepatotoxicity. Besides, increased neopterin levels may be also responsible for the development of irreversible hepatocellular damage (51). Gaonae Gaona L et al (15) reported that sulforaphane exhibits its hepatoprotective effects by protecting mitochondrial function and antioxidant enzymes and by preventing mitochondrial oxidative stress in the liver. There is no study in the literature showing the effects of sulforaphane on neopterin levels in experimental liver damage. In our study, hepatic neopterin levels were found to be lower in the treatment group with respect to the hepatotoxicity group. In addition, neopterin levels in this group decreased to the levels of the healthy control group. Which factors play a role in the progression of acetaminophen-induced liver damage is still uncertain. Focusing on various factors, new research studies are being carried out steadily. Macrophages as well as inflammatory cytokines were reported in various studies to increase in the liver in acetaminophen-induced hepatic damage $(48,49)$.

As a result, we obtained two important data. We can assert that sulforaphane administration in the acetaminophen-induced toxic hepatitis model is effective in reducing lipid peroxidation and oxidative stress-mediated liver damage. As an effective fighter against oxidative stress, sulforaphane provided similar neopterin levels as the control group, by preventing acetaminophene-induced hepatocellular damage. We expect positive results in case sulfora- phane is added in the treatment protocol of acetaminophen-induced hepatotoxicity. Optimal treatment strategies are not available yet, particularly in the prevention of toxic effects due to drug-induced toxic hepatitis, and we believe that further studies are needed on this topic. In addition, we believe neopterin can be used as a promising biomarker in the acetaminophen-induced hepatotoxicity.

\section{References}

1. Li L, Li W, Kim YH, Lee YW. Chlorella vulgaris extract ameliorates carbon tetrachloride-induced acute hepatic injury in mice. Exp Toxicol Pathol 2013; 65: 73-80.

2. Rajkapoor B, Venigopal Y, Anbu J, Harikrishnan N, Gobinath M, Ravichandran V. Protective effect of Phyllanthus polyphyllus on acetaminopheninduced hepatotoxicity in rats. Pak J Pharm Sci 2008; 21: 57-62.

3. Lee WM, Seremba E. Drug-induced liver disease. In: Yamada T(Ed). Textbook of Gastroenterology. Oxford: Blackwell Publishing, 2009, 2167-2184.

4. Aktas O, Eskiocak S, Ozgün GS, Yalcin O, Sut N. The effects of Lcarnitine on acetaminophen induced hepatotoxicity in rats. Turk J Biochem 2013; 38: 475-482.

5. Chung RT, Stravitz RT, Fontana RJ, Schiodt FV, Mehal WZ, Reddy KR, Lee WM. Pathogenesis of liver injury in acute liver failure. Gastroenterology 2012; 143: 7.

6. Hinson JA, Roberts DW, James LP. Mechanisms of acetaminopheninduced liver necrosis. Handb Exp Pharmacol 2010; 196: 369-405.

7. Halliwell B, Gutteridge JMC. Free Radicals in Biology and Medicine. Oxford: Oxford University Press, 2007, 55-79.

8. Chen W, Wang JB, Abnet CC, Dawsey SM, Fan JH, Yin LY, Yin J, Taylor PR, Qiao YL, Freedman ND. Association between C-reactive protein, incident liver cancer, and chronic liver disease mortality in the Linxian Nutrition Intervention Trials: a nested case-control study. Cancer Epidemiol Biomarkers Prev 2015; 24 (2): 386-392.

9. Cayci T, Akgül EO, Gumus S, Ucar E, Kurt YG, Tozkoparan E, Yaman H, Serdar MA, Cakir E, Bilgi C, Erbil MK, Deniz O. The Diagnostic Value of the Measurement of Neopterin Concentrations in Body Fluids for Pulmonary Tuberculosis. Turk J Biochem 2009; 34; 134-138.

10. Sucher R, Schroecksnadel K, Weiss G, Margreiter R, Fuchs D, Brandacher G. Neopterin, a prognostic marker in human malignancies. Cancer Lett 2010; 287: 13-22.

11. Demirbas S, Cakir E, Akgul EO, Seyrek M, Cayci T, Kurt YG, Uysal B, Aydin I, Kurt B, Yaman H, Erbil MK. Elevated serum neopterin levels in acetaminophen-induced liver injury. Environ Toxicol Pharmacol 2011; 31: 165-170.

12. Yaman H, Cakir E, Ozcan O, Yesilova Z, Ozcan A, Akgul EO, Erbil MK, Bagci S, Bilgi C, Dagalp K. Elevated urine neopterin levels in nonalcoholic steatohepatitis. Clin Biochem 2005; 38: 187-190.

13. Guerrero-Beltrán CE, Calderón-Oliver M, Pedraza-Chaverri J, Chirino YI. Protective effect of sulforaphane against oxidative stress: recent advances. Exp Toxicol Pathol 2012; 64: 503-508.

14. Glade MJ, Meguid MM. A Glance at... Broccoli, glucoraphanin, and sulforaphane. Nutrition 2015; 31: 1175-1178.

15. Gaona-Gaona L, Molina-Jijón E, Tapia E, Zazueta C, HernándezPando R, Calderón-Oliver M, Zarco-Márquez G, Pinzón E, PedrazaChaverri J. Protective effect of sulforaphane pretreatment against cisplatin-induced liver and mitochondrial oxidant damage in rats. Toxicology 2011; 286: 20-27. 
16. Gul H, Uysal B, Cakir E, Yaman H, Macit E, Yildirim AO, Eyi YE, Kaldirim U, Oztas E, Akgul EO, Cayci T, Ozler M, Topal T, Oter S, Korkmaz A, Toygar M, Demirbag S. The protective effects of ozone therapy in a rat model of acetaminophen-induced liver injury. Environ Toxicol Pharmacol 2012; 34: 81-86.

17. Ozcelik E, Uslu S, Erkasap N, Karimi H. Protective effect of chitosan treatment against acetaminophen-induced hepatotoxicity. Kaohsiung J Med Sci 2014; 30: 286-290.

18. Ohkawa H, Ohishi N, Yagi K. Assay for lipid peroxides in animal tissues by thiobarbituric acid reaction. Anal Biochem 1979; 95: 351-358.

19. Beutler E, Duron O, Kelly BM. Improved method for the determination of blood glutathione. J Lab Clin Med 1963; 61: 882-888.

20. Lowry OH, Rosebrough NJ, Farr AL, Randall RJ. Protein measurement with the Folin phenol reagent. J Biol Chem 1951; 193: 265-275.

21. Holt MP, Ju C. Mechanisms of drug-induced liver injury. AAPS J 2006; 8 (1): 48-54.

22. Arici S. Toxic Hepatitis. Pam Med J 2008; 1: 113-119.

23. Giannini EG, Testa R, Savarino V. Liver enzyme alteration: a guide for clinicians. CMAJ 2005; 172: 367-379.

24. Chalasani NP, Hayashi PH, Bonkovsky HL et al. ACG Clinical Guideline: The Diagnosis and Management of Idiosyncratic Drug-Induced Liver Injury. Am J Gastroenterol 2014; 109: 950-966;

25. Gardner CR, Gray JP, Joseph LB, Cervelli J, Bremer N, Kim Y, Mishin V, Laskin JD, Laskin DL. Potential role of caveolin-1 in acetaminophen-induced hepatotoxicity. Toxicol Appl Pharmacol 2010; 245: 36-46.

26. Noh JR, Kim YH, Hwang JH, Choi DH, Kim KS, Oh WK, Lee CH. Sulforaphane protects against acetaminophen-induced hepatotoxicity. Food Chem Toxicol 2015; 80: 193-200.

27. Xie W, Chen C, Jiang Z, Wang J, Melzig MF, Zhang X. Apocynum venetum Attenuates Acetaminophen-Induced Liver Injury in Mice. Am J Chin Med 2015; 43: 457-476.

28. Palani S, Raja S, Kumar BS. Hepatoprotective and antioxidant potential of Chloroxylon swietenia (Rutaceae) On Acetaminophen Induced toxicity in Male Albino Rats. Int J PharmTech Res 2010; 2: 162-170.

29. Jaeschke H, McGill MR, Williams CD, Ramachandran A. Current issues with acetaminophen hepatotoxicity-A clinically relevant model to test the efficacy of natural products. Life Sci 2011; 88: 737-745.

30. Oguz A, Kapan M, Kaplan I, Alabalik U, Ulger BV, Uslukaya O, Turkoglu A, Polat Y. The effects of sulforaphane on the liver and remote organ damage in hepatic ischemia-reperfusion model formed with pringle maneuver in rats. Int J Surg 2015; 18: 163-168.

31. Santana-Martínez RA, Galván-Arzáte $S$, Hernández-Pando R, Chánez-Cárdenas ME, Avila-Chávez E, López-Acosta G, PedrazaChaverrí J,Santamaría A, Maldonado PD. Sulforaphane reduces the alterations induced by quinolinic acid: modulation of glutathione levels. Neuroscience 2014; 272: 188-198.

32. Ballatori N, Krance SM, Notenboom S, Shi S, Tieu K, Hammond CL. Glutathione dysregulation and the etiology and progression of human diseases. Biol Chem 2009; 390: 191-214.

33. Baek SH, Park M, Suh JH, Choi HS. Protective effects of an extract of young radish (Raphanus sativus L.) cultivated with sulfur (sulfur-radish extract) and of sulforaphane on carbon tetrachloride-induced hepatotoxicity. Biosci Biotechnol Biochem 2008; 72: 1176-1182.
34. Zhao HD, Zhang F, Shen G, Li YB, Li YH, Jing HR et al. Sulforaphane protects liver injury induced by intestinal ischemia reperfusion through Nrf2-ARE pathway. World J Gastroenterol 2010; 16: 3002-3010.

35. Yilmaz S, Bahcelioglu IH. Lipid Peroxidation, Antioxidant Enzymes and Pyruvate Kinase Activity in Rats With Carbon Tetrachloride-Induced Cirrhosis. Turk J Vet Anim Sci 2000; 24: 25-28.

36. Yapar SB, Eskiocak S. The effect of alpha lipoic acid on hydrogen peroxide-induced lipid peroxidation in rat liver homogenates. Turk J Biochem 2014; 39: 317-327.

37. Halliwell B, Chirico S. Lipid peroxidation: its mechanism, measurement, and significance. Am J Clin Nutr 1993; 57: 715-725.

38. Aktas O, Eskiocak S, Ozgun GS, Yalcin O, Sut N. The effects of Lcarnitine on acetaminophen induced hepatotoxicity in rats. Turk J Biochem 2013; 38: 475-482.

39. Du K, McGill MR, Xie Y, Bajt ML, Jaeschke H. Resveratrol prevents protein nitration and release of endonucleases from mitochondria during acetaminophen hepatotoxicity. Food Chem Toxicol 2015; 81: 62-70.

40. Tien YH, Chen BH, Wang Hsu GS, Lin WT, Huang JH, Lu YF. Hepatoprotective and anti-oxidant activities of Glossogyne tenuifolia against acetaminophen-induced hepatotoxicity in mice. Am J Chin Med 2014; 42: $1385-1398$.

41. Terneus MV, Brown JM, Carpenter AB, Valentovic MA. Comparison of S-adenosyl-L-methionine (SAMe) and N-acetylcysteine (NAC) protective effects on hepatic damage when administered after acetaminophen overdose. Toxicology 2008; 244: 25-34.

42. Tolooei M, Mirzaei A. Effects of Pistacia Atlantica Extract on Erythrocyte Membrane Rigidity, Oxidative Stress, and Hepatotoxicity Induced by CCl4 in Rats. Glob J Health Sci 2015; 7: 46855.

43. Adeneye AA, Awodele O, Aiyeola SA, Benebo AS. Modulatory potentials of the aqueous stem bark extract of Mangifera indica on carbon tetrachloride-induced hepatotoxicity in rats. J Tradit Complement Med 2015; 5: 106-115.

44. Ozel KGS, Birdane YO. Antioxidants. Kocatepe Vet J 2014; 7: 41-52.

45. Murr C, Widner B, Wirleitner B, Fuchs D. Neopterin as a marker for immune system activation. Curr Drug Metab 2002; 3: 175-187.

46. Ertugrul I, Yolcu OF, Cesur S, Dagli U, Basar O, Yuksel I, Cakal B, Ozin YO, Ibis M, Ataseven H, Tunc B, Ulker A, Sasmaz N. Relationship between serum neopterin levels and inflammatory bowel diseases activity. Academic J Gastroenterol 2007; 6: 56-61.

47. Baydar T, Palabiyik S, Şahin G. Neopterin: Today’s Popular Biomarker? Review. Turkiye Klinikleri J Med Sci 2009; 29: 1280-1291.

48. Dambach DM, Watson LM, Gray KR, Durham SK, Laskin DL. Role of CCR2 in macrophage migration into the liver during acetaminopheninduced hepatotoxicity in the mouse. Hepatology 2002; 35: 1093-1103.

49. Cakir E, Akgul OE, Aydin I, Cayci T, Kurt YG, Onguru O, Aydin FN, Agilli M, Yaman H, Ersoz N, Bilgic S, Guven A, Turker T, Bilgi C, Erbil KM. The association between neopterin and acetaminophen-induced nephrotoxicity. Ren Fail 2010; 32: 740-746.

50. Fernandez E, Rodrigo L, Riestra S, Carcia S, Gutierrez F, Ocio G. Adenosine deaminase isoenzymes and neopterin in liver cirrhosis. J Clin Gastroenterol 2000; 30: 181-186.

51. Gulcan EM, Tirit I, Anil A, Adal E, Ozbay G. Serum neopterin levels in children with hepatitis-B-related chronic liver disease and its relationship to disease severity. World J Gastroenterol 2008; 14: 6840-6843.

Received March 8, 2017. Accepted April 4, 2017. 